(C) 1980. The Genetical Society of Great Britain

\title{
COMPETITIVE INTERACTIONS IN DROSOPHILA MELANOGASTER
}

\section{MONOCULTURES}

\author{
P. D. S. CALIGARI \\ Department of Genetics, University of Birmingham, P.O. Box 363 , \\ Birmingham B15 $2 T T$
}

Received 3.iii.80

\begin{abstract}
SUMMARY
A competition experiment with Drosophila melanogaster is reported which comprised a replacement series with two genotypes, together with pure cultures of both genotypes at the same densities as they appeared in the mixtures. The results for total numbers and weights make clear the need to separate the intergenotypic from the intragenotypic interactions if an unambiguous interpretation of the outcome of such mixtures is to be obtained. Further experiments were carried out to investigate the behaviour of pure cultures in order to gain insight into the relative simplicity or complexity of the intragenotypic interactions that needed to be taken into account when investigating mixtures. It was found that transformation of the measurements into percent survival and average weight per fly led to relations with density which were more nearly linear than the originals and, hence, were more readily amenable to analysis. Two main points emerged from the experiments. First, the relationship between the two transformed characters did not appear straightforward. Secondly, the intragenotypic interactions, particularly for percent survival, were complex and thus provided no support for assuming them to be simple when present in mixtures of genotypes.
\end{abstract}

\section{INTRODUCTION}

COMPETITION is commonly accepted to be one of the most, if not the most, important elements in natural selection whether natural populations or conditions of agricultural practice are being considered. Because of this interest it has received wide attention in the literature (witnessed, for example, by the references to competition in Harper, 1977) and yet the analysis even of simple mixtures appears not to be completely satisfactory, with different approaches used and varying amounts of information being obtained. Many studies have concentrated on mixtures of diverse genotypes, or even species, where phenotypic differences can be readily discerned and the fate of the component elements followed. Yet it is obvious that individuals of much closer relationship compete and indeed may do so more strongly than individuals of unlike genotype since the closer the genetical relationship between individuals the more similar are their requirements from the environment likely to be. Thus, as Mather (1969) points out, when we consider a simple mixture of two genotypes $\mathrm{X}$ and $\mathrm{Y}$ there are two classes of interaction to be considered. The first class is composed of two interactions being the effect of $\mathrm{X}$ on $\mathrm{Y}$ and its counterpart the effect of $Y$ on $X$. These are obviously intergenotypic interactions and are the ones that are usually explicitly discussed in studies of competition. The second class also is composed of two interactions being the effects of $\mathrm{X}$ on $\mathrm{X}$ and $\mathrm{Y}$ on $\mathrm{Y}$ which are, of course, intragenotypic interactions. Assumptions 
about the second class of interactions are made implicitly in most discussions of competition, but are commonly considered explicitly only in investigations of the effects of varying density in pure cultures of the components and in separate experiments from those investigating competition. Thus the two kinds are seldom considered together.

The need to take into account both sorts of interaction explicitly in the design and analysis of competition experiments can be seen from consideration of the first experiment described below.

\section{COMPETITION EXPERIMENT}

This experiment was carried out using two inbred lines of Drosophila melanogaster, namely Wellington (Well) and a line selected for high chaeta number $(6 \mathrm{CL})$. It was set up using counted numbers of eggs, as were all the experiments described in this investigation. This was achieved by collecting flies of the two genotypes separately and keeping them for a few days at low density to ensure successful mating. Flies within a genotype were then massed overnight at $28^{\circ} \mathrm{C}$ before being allowed to lay for a few hours on charcoal-darkened starch sticks. The required number of eggs were then picked off the starch stick with a needle and placed on the surfaces of previously prepared tubes. The medium in the tubes consisted of $5 \mathrm{ml}$ of 2 per cent agar onto the surface of which had been dispensed $0.5 \mathrm{ml}$ of yeast suspension (the suspension being made up of $12 \mathrm{gm}$ of dried baker's yeast in $100 \mathrm{ml}$ of distilled water). Thus the food source available to the developing larvae was the measured amount of yeast.

The experimental design employed was that of a replacement series (de Wit, 1960) of mixtures with a total density of 96 eggs but also included were the pure or monocultures of the two genotypes at the same densities as they were used as components in the mixtures, a design Seaton and Antonovics (1967) used. The experiment consisted of the following cultures:

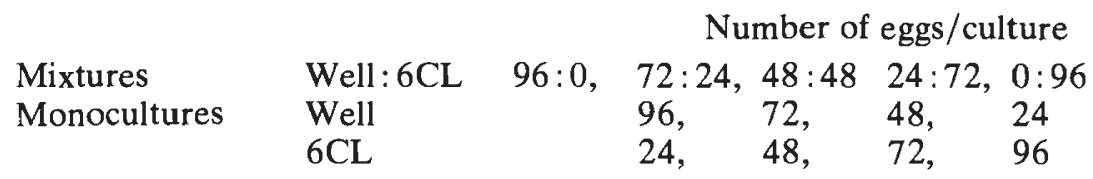

The 96:0 and 0:96 mixtures are, of course, the same as 96 eggs of the pure cultures. The whole experiment was set up in quadruplicate. All cultures were incubated at $25^{\circ} \mathrm{C}$ and on each day for a week from the first sign of emergence adult numbers and their total weights, in mgms, were recorded. The score for each character from each culture was obtained by summing the observations over days. The results, averaged over the four replicates of this experiment, are presented in graphical form for total numbers in fig. 1a and total weights in fig. $1 \mathrm{~b}$.

Consider first the results from the mixtures which are shown as solid lines in fig. 1. For both numbers and weights the customary interpretation of the shapes of the relations would suggest that the effect of Well on 6CL was greater than the effect of $6 \mathrm{CL}$ on itself while the effect of $6 \mathrm{CL}$ on Well was less than Well on itself. In this experiment, however, it is possible to examine the effect of a genotype on itself by considering the responses of the 
(a)

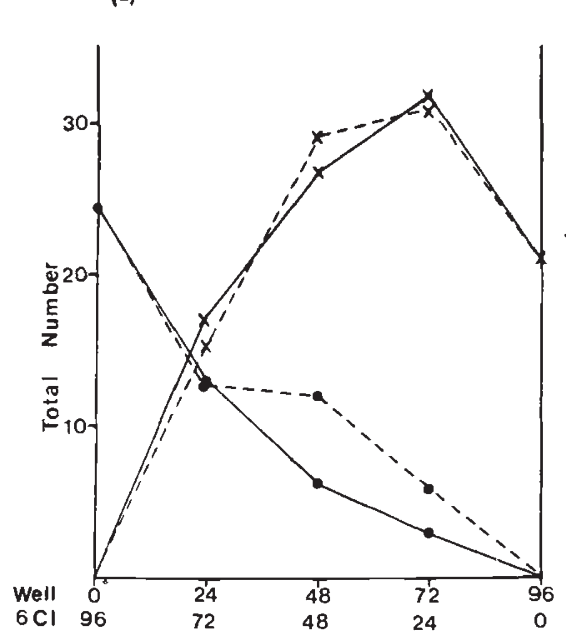

(b)

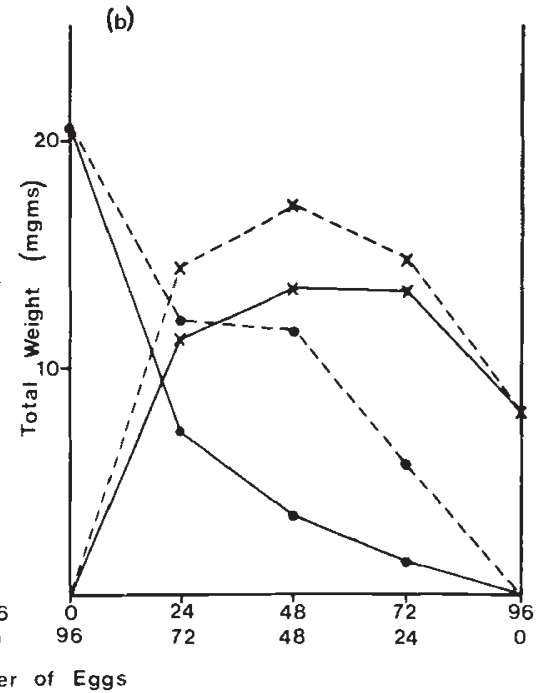

FIG. 1.-Density replacement series of two inbred lines namely Well $(x)$ and 6CL $(O)$. Mixtures are represented by solid lines and monocultures by dashed lines. Each point represents the average of four replicate cultures. (a) shows the results for total numbers of adult flies emerging and (b) those for total weight of such flies.

monocultures shown as dashed lines. Looking first at the results for total numbers given in fig. $1 \mathrm{a}$ it can be seen that the responses of both genotypes are very much the same whether in pure or mixed cultures. It could therefore be argued that in the mixtures, the two genotypes are having little effect on one another, i.e., there is little intergenotypic interaction, while the effects on self, i.e., intragenotypic interactions, are mainly responsible for the observed relations. In other words the outcome for either genotype from a mixture is almost completely dependent on the density of its own genotype present and not on the other genotype. It should be noted that, in fact, only if neither inter- nor intra-genotypic interaction was present would the pure and mixed culture responses be completely linear.

Turning attention now to the results for total weight of flies, given in fig. $1 \mathrm{~b}$, it can be seen that the pure and mixed culture responses do appear different indicating the presence of intergenotypic interactions. Again, as for total numbers, the changes in total weight in pure cultures are not simply linearly related to the initial number of eggs and therefore indicate the presence of intragenotypic interactions. Taking the pure and mixed culture responses in to account it would appear that, as we surmised earlier, the yield in weight of $6 \mathrm{CL}$ is reduced by the presence of Well but it is now clear that the weight of Well is also reduced by $6 \mathrm{CL}$.

This experiment makes clear that if unambiguous information is to be obtained concerning the interactions within mixtures of genotypes it is necessary to be able to detect both the intra- and the inter-genotype interactions. Competition experiments therefore need to be carried out and analysed in such a way as to provide a measurement of the two types of interaction separately, allowing the use of the intragenotypic interactions as a yardstick against which to assess the intergenotypic interactions. With 
these considerations in mind the first part of the investigation, the part with which this paper is concerned, was to examine the behaviour of monocultures of Drosophila melanogaster under defined conditions for both numbers of adults produced and weight of flies over a range of densities. The relative simplicity or complexity of the behaviour of such monocultures would then provide a key to the approach needed to analyse mixtures of genotypes.

\section{MONOCULTURE EXPERIMENTS}

\section{(i) The genotypes}

In the experiment described above the two genotypes used were both highly inbred lines which, as commonly is the case between true breeding lines, differed in the hatchability of their eggs. In uncrowded conditions and a plentiful food supply Well had a hatchability of around 80 per cent while 6CL was nearer 30 per cent, this meant that inclusion of equal numbers of eggs of the two genotypes did not give a corresponding equality in the numbers of competing larvae. To avoid such large discrepancies in the number of competing individuals at this stage of the investigation, it was decided to cross inbred lines in pairs and hence have $F_{1}$ individuals competing. Such crosses appeared to have much more uniform hatchabilities of between 70 and 80 per cent while still retaining the obvious advantages of being able repeatably to produce the same genotype in large numbers on different occasions.

Although in this paper interest centres on the results from pure cultures, they are preliminary to the investigation of mixtures of genotypes where it would be an obvious advantage to be able to recognise the genotypes of the emerging adult flies. Therefore the mutant allele $y^{2}$ (yellow body colour) was introduced into some of the inbred lines maintained in the Genetics Department at Birmingham. This was achieved using procedures that are standard with Drosophila melanogaster. The stock used to provide the $y^{2}$ allele was one containing $y^{2} w^{a} c v v f$. Since $y^{2}$ is very close to the left end of the $\mathrm{X}$ chromosome and $w^{a}$ (apricot eye colour) is only 1.5 map units from $y^{2}$, recombinants can be obtained which consist of the intact X chromosome of the inbred line required with only the small portion of the left end containing $y^{2}$ having been substituted. The IInd and IIIrd chromosomes of the inbred line were retained intact against marked inversions, the $Y$ chromosome was always derived from the marked stock and the small IVth chromosome was left uncontrolled. Thus the four inbred lines which formed the basis of the present experiment, namely Well, 6CL, Sam (Samarkand) and Ed (Edinburgh) had lines which should be direct genetical counterparts but containing the $y^{2}$ allele, i.e., $y^{2}$ Well, $y^{2} 6 \mathrm{CL}, y^{2}$ Sam and $y^{2} \mathrm{Ed}$. These eight lines were then crossed in pairs to produce the four $F_{1}$ genotypes, which were to be investigated, as follows: (A) $6 \mathrm{CL} \times$ Well, $\left(\mathrm{A}^{\prime}\right) y^{2} 6 \mathrm{CL} \times$ $y^{2}$ Well, (B) Ed $\times$ Sam and $\left(\mathrm{B}^{\prime}\right) y^{2} \mathrm{Ed} \times y^{2}$ Sam. Obviously any failure of the inversions to suppress recombination during the production of the $y^{2}$ lines, any effect of $y^{2}$ itself (or a very closely associated region) or any effects traceable to the IVth chromosome, which was left uncontrolled, will tend to make A differ from $A^{\prime}$ and $B$ differ from $B^{\prime}$. Such effects, in as far as they cause differences in intragenotypic interactions, can be tested for by raising the monocultures of the inbred and $y^{2}$ crosses at varying densities in a single 
randomised experiment. A later paper, dealing specifically with mixtures of genotypes, will present data which allow more extensive testing of any differences produced in the competitive ability of these genotypes in terms of their inter-, as well as intra-genotypic interactions.

\section{(ii) Varying density}

Eggs of the four crosses were counted into tubes, as described in the earlier experiment, to produce monocultures of each genotype with the number of eggs/tube given in table 1 , where the numbers of replicates are also shown. The total number of flies emerging from each culture and their total weight were recorded as in the earlier experiment and the results, averaged over replicates, are presented in graphical form in fig. $2 a$ and $b$ respectively.

TABLE 1

The number of eggs/tube and the number of replicates

$\begin{array}{lrrrrrrrrrrr}\text { Eggs/tube } & 2 & 8 & 16 & 20 & 24 & 36 & 48 & 72 & 96 & 144 & 192 \\ \text { No. of replicates } & 8 & 8 & 6 & 6 & 4 & 4 & 4 & 4 & 4 & 2 & 2\end{array}$

The first point that is clear from fig. 2 is that total numbers and weights increase with increasing density up to a maximum after which the yields for both characters decrease. In other words at the lower densities increasing the number of eggs has a greater effect on yield than any comcomitant decrease due to increased competition. After a certain density, however, competitive effects become predominant and subsequently the yield falls. In fact other experiments with these genotypes showed that at higher densities no adults emerged at all.

Secondly, it can be seen that the maximum yield for total weight is reached at a lower density than for numbers. For $A$ and $A^{\prime}$ the maximum total weight is produced at a density near 96 eggs/tube while the maximum total numbers are not produced until a density of about 144 eggs/tube is reached. Similarly for B and B' the maxima are around 72 and 96 eggs/tube for weights and numbers respectively. This comparison leads to the third point which concerns the differences between the four $F_{1}$ genotypes. It is clear that differences are present between the four genotypes and that apart from very low or high densities the differences between the two sets, the one being $\mathrm{A}$ and $\mathrm{A}^{\prime}$ and the other $\mathrm{B}$ and $\mathrm{B}^{\prime}$, are considerably larger than those within sets. In other words the results suggest that while the insertion of the $y^{2}$ allele has altered the genetical differences between the sets as far as intragenotypic interactions affecting the two characters are concerned it has not done so to any serious extent.

The data as they stand are, however, not in the most convenient form for proceeding further. In the first place the responses are clearly curvilinear and although this can, of course, be handled statistically the analysis is more cumbersome and the interpretation less readily apparent than with a linear relationship. This is particularly true when it is desired not only to compare the monoculture responses with each other but also, ultimately, with the results from mixtures. Secondly, the characters are not completely independent of one another since total weight may be regarded as total 


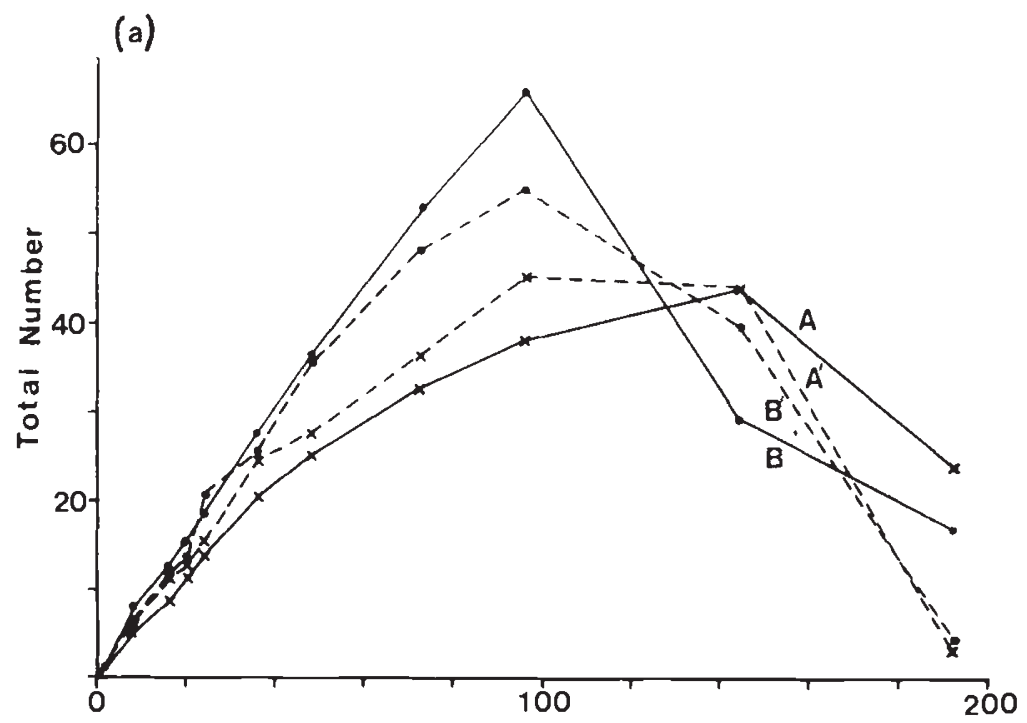

(b)

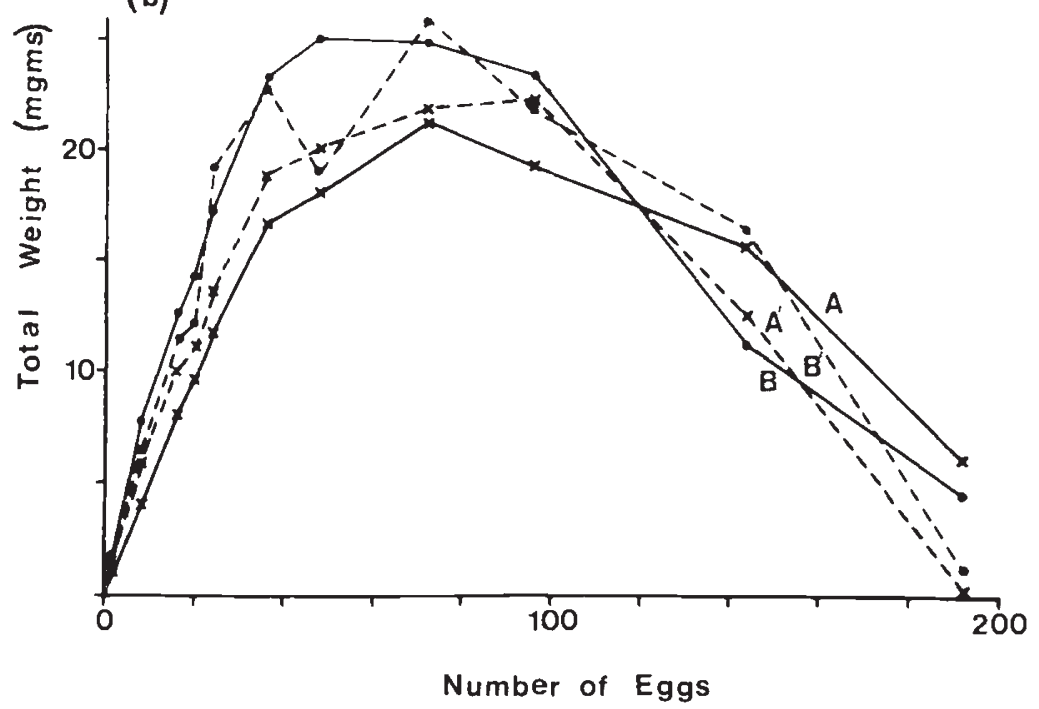

FIG. 2.-Monocultures started with different numbers of eggs for two $F_{1}$ 's, $A$ and $B$. Each is represented by its wild-type (solid line) and $y^{2}$ equivalent (dashed line) as detailed in the text. Presented in (a) are the data for total numbers emerging and (b) the total weight of such flies.

numbers multiplied by average weight per fly and thus the responses of the two characters will tend to appear similar.

To rectify these shortcomings it was decided to transform the two original measurements into: (i) the percentage survival obtained as

$$
\frac{\text { number of adults emerging }}{\text { number of eggs put in }} \times 100
$$


(a)

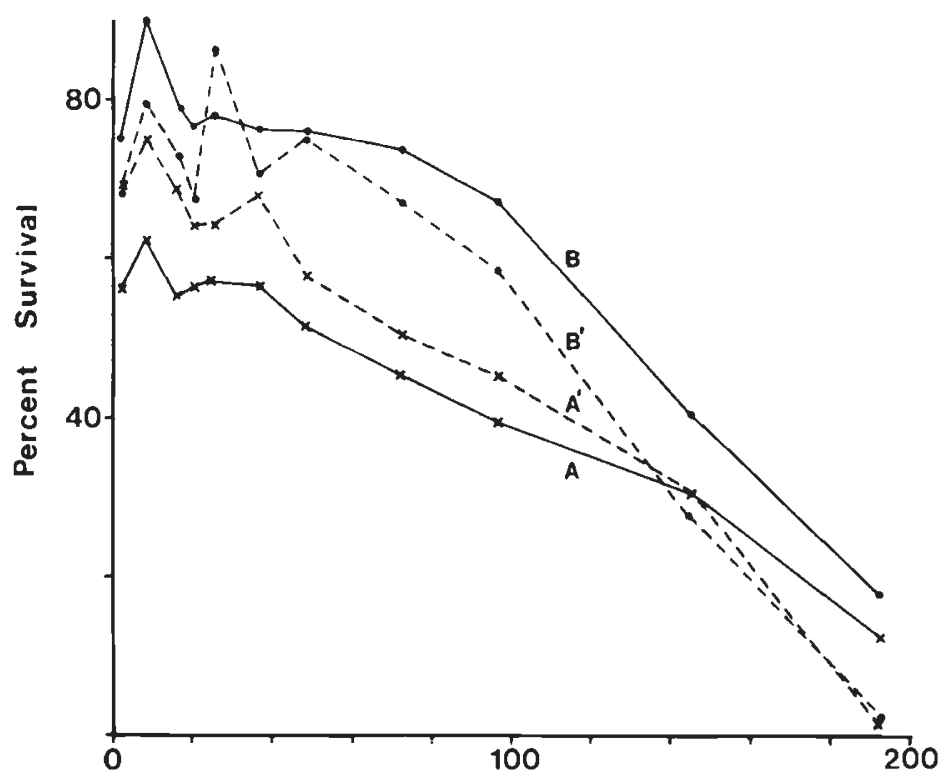

(b)

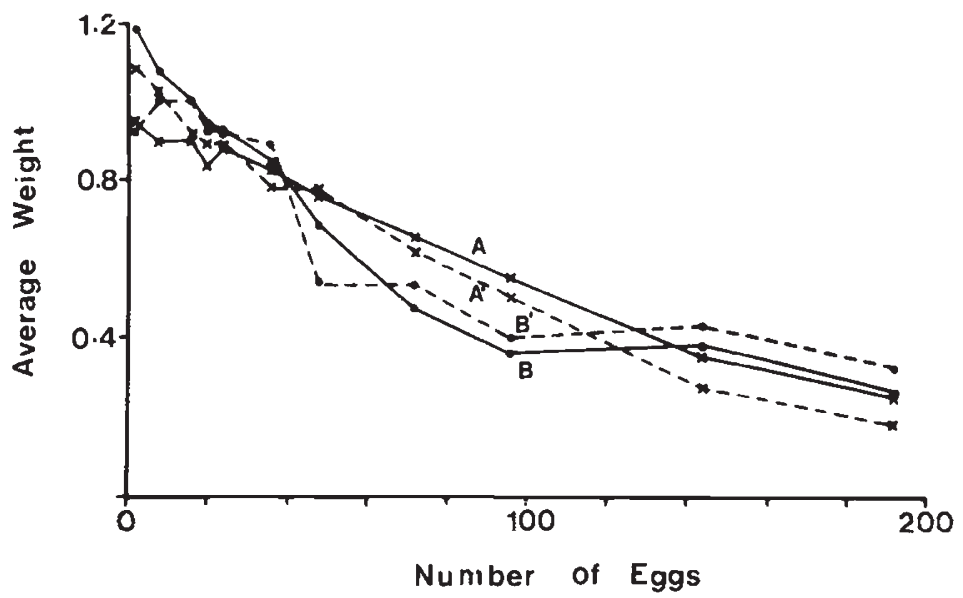

FIG. 3.-The observations presented in fig. 2 transformed in (a) to percent survival and (b) average weight per fly.

(which can, of course, be further transformed into angles for analysis) which is equivalent to the probability of a single egg giving rise to an adult fly, and (ii) the average weight per fly given by

$$
\frac{\text { Total weight of emerging flies }}{\text { Total numbers of emerging flies }}
$$

in other words the expected weight of any fly that does survive. If the data for each culture of the present experiment are transformed in this way and averaged over replicates fig. $3 a$ and $b$ are obtained. 
The first obvious feature of the figures is that the responses of the transformed characters approximate much more nearly to linearity than did those on the original scales, shown in fig. 2, particularly if the results for percent survival (fig. $3 \mathrm{a}$ ) at low density are left aside. The second point of interest is that on the transformed scales there is again no evidence of the introduction of the $y^{2}$ allele having any major effect except perhaps on percent survival at the highest density.

The third point concerns the responses in percent survival at low density which, as already observed, depart from the general linear response. Over these densities the percent survival actually increases from the lowest density up to a maximum after which survival begins to decrease fairly regularly. In other words at low density the chance of a fly surviving increases as density increases and so, of course, indicates the presence of cooperation. This is in good agreement with the earlier results of Kearsey (1965). Clearly cooperation could have been detected with the original data of fig. 2 but the transformation has made its detection simpler. There is no similar evidence for cooperation affecting average weight, so suggesting that the two characters are not responding in exactly the same way to low density.

Fourthly, when comparing the two $\mathrm{F}_{1}$ genotypes, $\mathrm{A}$ and $\mathrm{A}^{\prime}$ on the one hand and $\mathrm{B}$ and $\mathrm{B}^{\prime}$ on the other, it appears that for percent survival not only do they reach different maxima at low density but they also decrease at different rates over the higher densities. Conversely, for average weights the responses are much more alike, giving no clear evidence of differences between the genotypes.

Generally, the experimental results suggest that over the densities at which competition is occurring, transforming the data into percent survival and average weight per fly produces relations which at least approximate to linearity. They also demonstrate the possibility of detecting genetical differences affecting these two characters which behave to some extent independently in their responses to density.

\section{(iii) Different food levels}

A third experiment was undertaken to investigate the effect of varying the amount of food available per larva at a range of different egg densities. The experimental technique was as in the previous experiments with again $0.5 \mathrm{ml}$ of yeast suspension dispensed into each tube but in this experiment the concentration of the suspension was varied. Three levels of yeast were in fact used namely, Normal (N) which consisted, as before, of $12 \mathrm{gm}$ of dried yeast suspended in $100 \mathrm{ml}$ of distilled water, Half $(\mathrm{H})$ being $6 \mathrm{gm} / 100 \mathrm{ml}$ and Double (D) $24 \mathrm{gm} / 100 \mathrm{ml}$. Two $F_{1}$ genotypes were studied and as before both were present as wild-type as well as the $y^{2}$ counterparts. There were, therefore, four $\mathrm{F}_{1}$ 's present, the $\mathrm{B}$ and $\mathrm{B}^{\prime}$ of the last experiment and $\mathrm{C}$ and $C^{\prime}$, a cross between two lines derived by inbreeding from the Texas population (Texas 28 and Texas 27) and its $y^{2}$ equivalent. The densities investigated and number of replicates used were the same for each of the yeast levels and are shown in table 2 . The whole experiment was carried out on two separate occasions.

Analyses of variance were carried out by taking means of the replicates within a density and food level as the basic data and using the differences between occasions to provide a measure of error variance. The analysis of 
TABLE 2

The number of eggs/tube and the number of replicates used at each of the three yeast levels Half, $(H)$, Normal $(N)$ and Double $(D)$

Eggs/tube

No. of replicates

$\begin{array}{rrrrrr}30 & 60 & 90 & 120 & 150 & 180 \\ 6 & 3 & 2 & 1 & 1 & 1\end{array}$

percent survival was carried out on the data transformed to angles and hence a theoretical error variance could be calculated for it. For average weight the analysis was performed on the data directly. However, since at the higher densities on $\mathrm{H}$, some cultures, although active, produced no survivors an average weight could not be estimated and therefore the analysis of this character was confined to the results of densities $30,60,90$ and 120 over all food levels.

A complete analysis of variance of either character would provide measures of the effect of density, food levels and genotypes as well as, of course, the various interactions between them. Indeed the analysis could be further elaborated by the partition of these items into various meaningful comparisons. For instance, densities can be separated into items displaying the effects of linear, quadratic or even higher orders of regression. Genotypes can be separated into variation attributable to the difference between the two $\mathrm{F}_{1}$ 's, the effects of introducing the $y^{2}$ allele and, of course, their interaction. However, in the present experiment the principal concern is with the effects of density and food level and, therefore, the analyses given in table 3 have been simplified to reflect this. Table 3 a shows the analysis for percent survival and $3 \mathrm{~b}$ that for average weight. In the table the pooled

\section{TABLE 3}

The analyses of variance of the results are given for (a) percent survival (in angles) and (b) average weight. For each character the appropriate pooled genotypic effects have been taken as the estimate of error for testing the other items

\[ (a) \]
Densities $(\mathrm{D})$
$\left\{\begin{array}{l}\text { linear } \\ \text { rest }\end{array}\right.$
Food levels $(\mathrm{F})$
$\mathrm{F} \times \mathrm{D}$
$\left\{\begin{array}{l}\mathrm{F} \times \text { linear } \\ \mathrm{F} \times \text { rest }\end{array}\right.$

Pooled genotype effects

Occasions

Theoretical error

\begin{tabular}{|c|c|c|c|c|}
\hline SS & df & M.S. & $\chi^{2}$ & $\mathrm{P}$ \\
\hline $6253 \cdot 6$ & 5 & $1250 \cdot 7$ & $90 \cdot 8$ & $<0.001$ \\
\hline $6183 \cdot 2$ & 1 & $6183 \cdot 2$ & $89 \cdot 7$ & $<0.001$ \\
\hline $70 \cdot 4$ & 4 & $17 \cdot 6$ & $1 \cdot 0$ & N.S. \\
\hline 2796.4 & 2 & $1398 \cdot 2$ & $40 \cdot 6$ & $<0.001$ \\
\hline $5991 \cdot 0$ & 10 & $599 \cdot 1$ & $87 \cdot 0$ & $<0.001$ \\
\hline 5468.4 & 2 & $2734 \cdot 2$ & $79 \cdot 4$ & $<0.001$ \\
\hline 522.6 & 8 & $65 \cdot 3$ & $7 \cdot 6$ & N.S. \\
\hline $3720 \cdot 1$ & 54 & 68.9 & & \\
\hline $1274 \cdot 5$ & 72 & $17 \cdot 7$ & & \\
\hline & & $5 \cdot 1$ & & \\
\hline
\end{tabular}

(b)

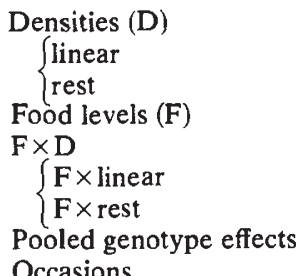

$$
\begin{aligned}
164.3 & <0.001 \\
160.6 & <0.001 \\
3.7 & \text { N.S. } \\
407.6 & <0.001 \\
101.1 & <0.001 \\
83.7 & <0.001 \\
17.4 & <0.001
\end{aligned}
$$


genotype effects for each character have been used as the error against which all other items have been tested. In other words, the test was whether there was variation in the effects of density, food level and their interaction over and above the variation due to genotypes. It should be emphasised, however, that in the full analysis of both characters some of the line items, including the comparisons of wild-type with $y^{2}$, were significant when tested against the occasions mean square (M.S.). In fact in both cases the pooled genotype effects are larger than the occasions M.S. and in the case of percent survival significantly so. However, none of the M.S.'s of the genotype effects or the various genotype interactions was as large as those for density, food level or their interaction as can be judged from consideration of the appropriate sums of squares (S.S.) in table 3. The analyses presented make clear that for both characters changes in density and food level and their

(a)

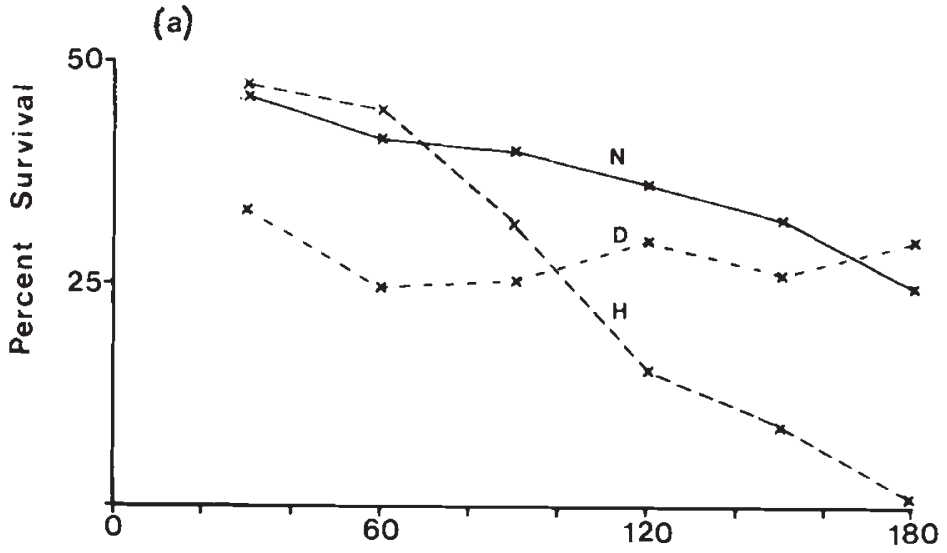

(b)

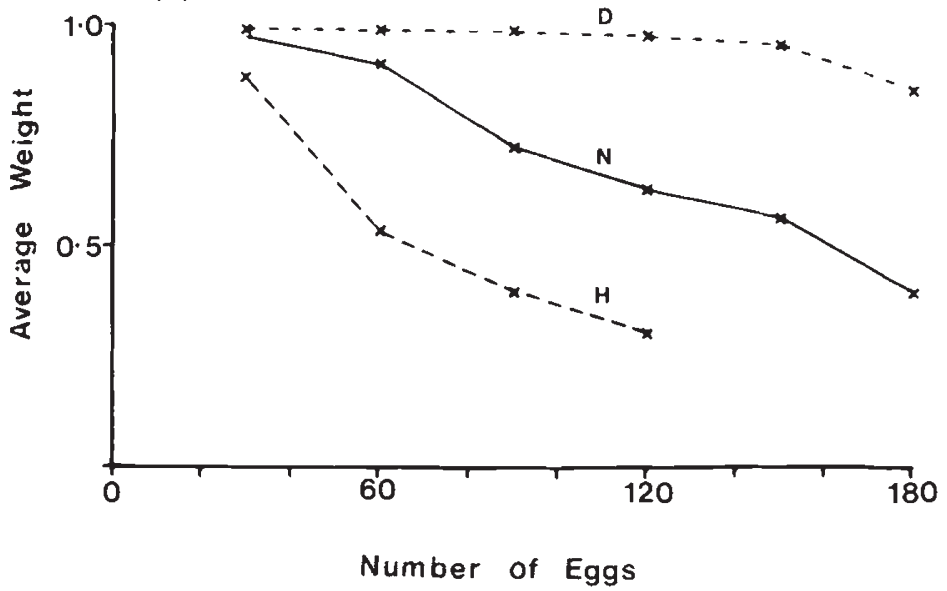

FIG. 4.-The results from varying numbers of initial eggs cultured on three different food levels, averaged over genotypes, replicates and occasions (a) shows the results for percent survival where each point is subject to a s.e. \pm 0.30 and (b) those for average weight per fly with a s.e. $\pm 0 \cdot 03$. Solid lines represent the results from Normal food level $(\mathrm{N})$, heavy dashes for Half food $(\mathrm{H})$ and light dashes for Double food (D). 
interaction are significantly greater than the genotypic variation. Partitioning the densities items shows that the linear component of the response is by far the largest. The interaction term shows the response to remain linear at the different food levels, but the slopes of the regression lines to vary.

Since, as has already been noted, we are considering genotypes as replicates for our present purpose, we can average over them and plot the means for the characters separately against density for each of the three food levels. Fig. 4a shows the results for percent survival and $4 \mathrm{~b}$ those for average weight. Each of the points on the graphs is the mean of 8 observations and will be subject to a variance of one eighth of the genotypic variance. A standard error (s.e.) of \pm 2.93 in angles corresponding in percent to a little under \pm 0.3 can therefore be attached to the means in fig. $4 \mathrm{a}$ while those in $4 \mathrm{~b}$ will have a s.e. of \pm 0.03 . As a companion to fig. $4 \mathrm{a}$, fig. 5 has been drawn showing percent survival, averaged over food levels, plotted against density for each of the genotypes separately. Comparison of the two figures emphasises that the differences between the genotypes, though formally significant, is very much smaller than those between food levels.

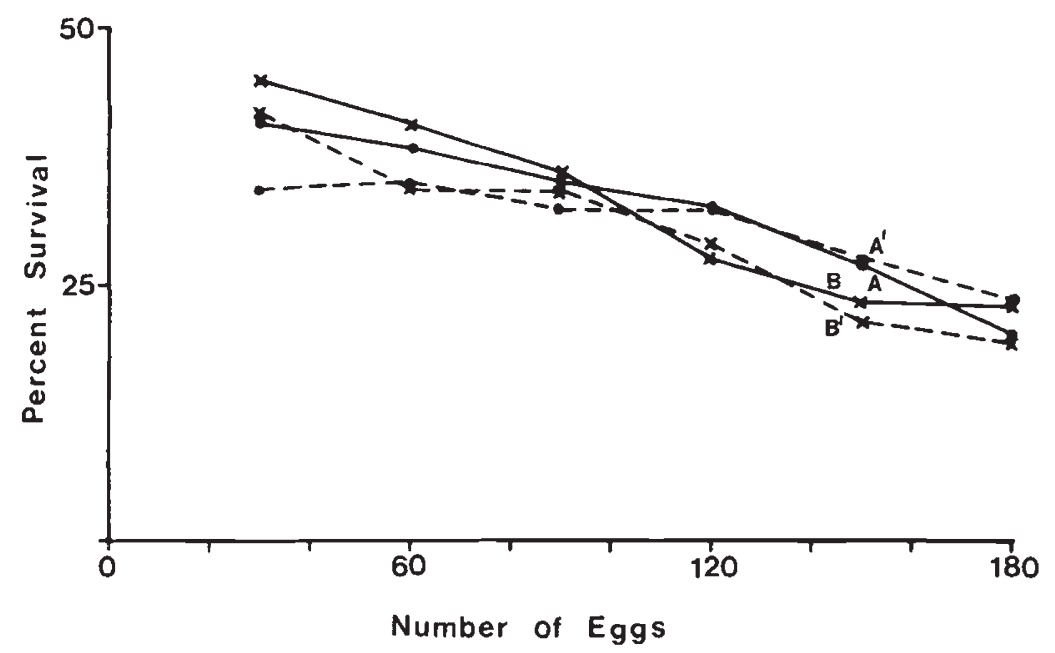

FIG. 5.-The results for percent survival from varying numbers of initial eggs for two $F_{1}$ genotypes each represented as wild-type (solid lines) and $y^{2}$ (dashed line), averaged over the three food levels as well as replicates and occasions (cf. fig. 4(a)).

Returning to fig. $4 \mathrm{~b}$, it can be seen that on food level $\mathrm{H}$ the average weight of the flies decreases steadily from the lowest density onwards. At food level $\mathrm{D}$ there is little change over most of the range though at the highest density the average weight starts decreasing. The middle food level, $\mathrm{N}$, produces an intermediate response with only a slight fall from density 30 to 60 but thereafter giving a steady decrease at much the same rate as that observed for $\mathrm{H}$. In other words the results are consistent with the hypothesis of the different food levels simply altering the density at which the effects of competition are expressed but not altering their magnitude.

In contrast to average weight the graph for percent survival (fig. 4a) appears less straightforward. At densities of 30 and 60 eggs, half food $(\mathrm{H})$ produces the highest percent survival, normal $(\mathrm{N})$ the next highest and 
double food (D) the lowest. Not until the highest density, i.e., 180 eggs, is reached does the order become reversed. It is clear that on $\mathrm{D}$ the percent survival does not change very greatly over the range of densities studied but the overall relatively low level of survival is surprising. It is possible that at higher food levels a higher density of eggs is required to reach the cooperative peak that was observed on normal food in fig. 3a. Two factors, however, argue against this being the explanation of the present results. First, there is no real indication of any rise in percent survival such as could be taken to indicate cooperation even at the highest densities and it would seem unlikely that cooperation and competition exactly balanced each other over the whole range of densities. Secondly, results from many other experiments carried out during this investigation together with those of Kearsey (loc. cit.) have consistently found the cooperative peak to occur at low density, commonly less than 20 eggs/tube, certainly at very much lower densities than $180 \mathrm{eggs} /$ tube. The other two food levels appear to give fairly steady decreases in survival, above a density of 60 eggs, but they decrease at apparently different rates. In general it would seem that there is not a simple relationship between food level and percent survival in that percent survival of, for instance, $2 x$ eggs on double unit food is not readily predictable from the results of $x$ eggs on single unit food.

The results of this experiment bring out two points. First, the two characters do not respond in the same manner to changes of density at the three food levels. Secondly, at least for percent survival, competitive interactions must depend on more than just the amount of food available per individual.

\section{Conclusions}

The experiments reported in this paper form part of a more extensive investigation into the competitive interactions between individuals. Other experiments carried out on pure cultures, but not reported here, include investigating the effects of killed as opposed to live yeast, the adding of anti-fungal agents to the medium and the differences produced by altering the surface area of the available food. These further experiments, although of interest in their own right, concern us now chieffy because of the support they lend to the two main conclusions that can be drawn from the experiments reported here. First, the relationship between the two characters studied, percent survival and average weight, is not simple. This is not to say that no such relationship exists since it has been suggested previously that the variation in such factors as larval feeding rates, critical weights for pupation, efficiency of food conversion and post-critical feeding (Burnet $e t$ al., 1977; Royes and Robertson, 1964 and Robertson, 1964) can affect the outcome of competition in Drosophila. However, any formula connecting percent survival and average weight would be complex and, therefore, probably of restricted use in the present context. Thus for practical purposes it would seem reasonable to treat them as separate characters. Secondly, the experiments clearly bring out the complexity of the intragenotypic interactions that are present even in pure cultures of Drosophila, under the relatively simple conditions of this investigation. There could be no justification for assuming that such interactions will be any simpler when they are present in mixed cultures of genotypes. 
Therefore, if an unambiguous interpretation of genetically heterogeneous cultures is to be obtained it is necessary to design and analyse competition experiments so as to allow the different interactions to be separated and quantified in such a way as to enable the intergenotypic interactions be be measured against the intragenotypic as a yardstick.

Acknowledgments. - I gratefully acknowledge the continuing help and advice of Professor Sir Kenneth Mather. Financial support from the Agricultural Research Council made this work possible.

\section{REFERENCES}

BURNET, B., SEWELL, D., AND BOS, M. 1977. Genetic analysis of larval feeding behaviour in Drosophila melanogaster. II. Growth relations and competition between selected lines. Genet. Res., Camb. 30, 149-161.

DE WIT, C. T. 1960. On Competition. Versl. Landbouwk. Onderz. Ned., 66, 1-82.

HAR PeR, J. L. 1977. Population Biology of Plants. Academic Press, London.

KEARSEY, M. J. 1965. Cooperation among larvae of a wild-type strain of Drosophila melanogaster. Heredity, 20, 309-312.

MATHER, K. 1969. Selection through competition. Heredity, 24, 529-540.

ROBERTSON, F. W. 1964. The ecological genetics of growth in Drosophila. 7. The role of canalization in the stability of growth relations. Genet. Res., Camb. 5, 107-126.

ROYES, W. V., AND ROBERTSON, F. W. 1964. The nutritional requirements and growth relations of different species of Drosophila. J. Exp. Zoo., 156, 105-136.

SEATON, A. P. C., AND ANTONOVICS. J. 1967. Population inter-relationships. I. Evolution in mixtures of Drosophila mutants. Heredity, 22, 19-33. 